\title{
Improved integral images compression based on multi-view extraction
}

\author{
Antoine Dricot ${ }^{\mathrm{a}, \mathrm{b}}$, Joel Jung ${ }^{\mathrm{a}}$, Marco Cagnazzo ${ }^{\mathrm{b}}$, Béatrice Pesquet $^{\mathrm{b}}$, and Frédéric Dufaux ${ }^{\mathrm{b}}$

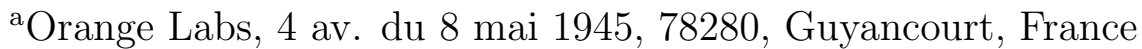 \\ bLTCI, CNRS, Télécom Paristech, Université Paris-Saclay, 75013, Paris, France
}

\begin{abstract}
Integral imaging is a technology based on plenoptic photography that captures and samples the light-field of a scene through a micro-lens array. It provides views of the scene from several angles and therefore is foreseen as a key technology for future immersive video applications. However, integral images have a large resolution and a structure based on micro-images which is challenging to encode. A compression scheme for integral images based on view extraction has previously been proposed, with average BD-rate gains of $15.7 \%$ (up to $31.3 \%$ ) reported over HEVC when using one single extracted view. As the efficiency of the scheme depends on a tradeoff between the bitrate required to encode the view and the quality of the image reconstructed from the view, it is proposed to increase the number of extracted views. Several configurations are tested with different positions and different number of extracted views. Compression efficiency is increased with average BD-rate gains of $22.2 \%$ (up to $31.1 \%$ ) reported over the HEVC anchor, with a realistic runtime increase.
\end{abstract}

Keywords: Integral imaging, plenoptic, multi-view extraction, compression, 3D-HEVC

\section{INTRODUCTION}

Improvements in video technologies aim to provide immersive viewing experiences. However, current 3D video technologies are still very limited. In stereoscopic 3D, the glasses cause discomfort and the conflict between the eye accommodation and convergence distances is reported to cause headaches and eyestrain. Auto-stereoscopic display systems with more than two views provide unrealistic perception stimuli, and have restricted viewing zones (sweet spots) that prevent smooth motion parallax, which is a key element in the perception of depth [1].

Integral imaging is based on plenoptic photography [2]. It samples the light-field of a scene [3][4] and provides several views of that scene from different angles. Light-field has recently been drawing a lot of interest from the experts in the domain, and is foreseen as a key technology for future immersive applications (e.g. such as immersive telepresence) [5][6]. However, integral images have a large resolution in order to provide a large number of viewpoint images with a sufficient resolution. Moreover, the Micro-Images (MIs) based structure is challenging to encode.

New efficient coding technologies are required to handle these characteristics. In [7] we proposed an original and efficient compression scheme to encode integral images. This scheme takes advantages of the view extraction process to reconstruct a reliable predictor and creates a residual integral image that is encoded. In this paper we propose to further study the impact of the configuration of the view extraction and to improve the performance of the scheme using multi-view extraction and multi-view compression.

This paper is organized as follows. In Section 2, an overview of view extraction methods is given, and stateof-the-art methods to encode integral imaging content are presented. Our view extraction based compression scheme is described in Section 3.1 and improvements are proposed in Section 3.2. Experimental conditions and experimental results are given in Section 4 and Section 5 respectively. Conclusions are drawn in Section 6.

Further author information: (Send correspondence to A.D.)

A.D.: antoine.dricot@orange.com

J.J.: joelb.jung@orange.com

M.C.: cagnazzo@telecom-paristech.fr

B.P.: pesquet@telecom-paristech.fr

F.D.: dufaux@telecom-paristech.fr 

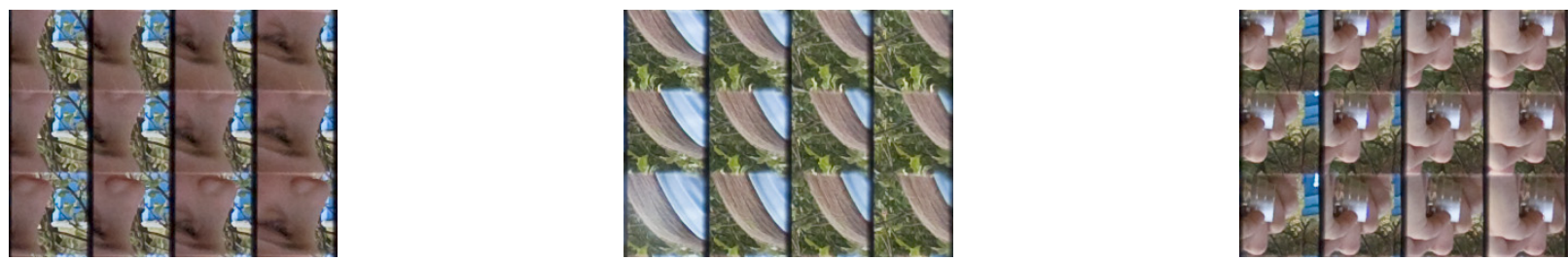

Figure 1. Close-up on different Micro-Images (image: Laura [8])

\section{RELATED WORK}

\subsection{State-of-the-art view extraction methods}

Integral imaging capture devices (e.g. plenoptic cameras provided by Lytro [9] or Raytrix [10]) samples the lightfield using a lenticular array, composed of many micro-lenses. Each micro-lens creates one MI in the resulting integral image, which contains light information coming from several angles of view, as illustrated in Fig. 1.

An overview of several view extraction methods is given in [11]. The basic method copies one patch (a square zone of pixels) from each MI into the extracted view, as illustrated in Figure 2. This method uses the characteristics of the focused plenoptic camera [12] for which there are both angular and spatial information within one MI. The relative position of the patch within the MI determines the angle of view. A more basic method extracts only one pixel per MI, (i.e. a patch of size $1 \times 1$ ). The depth plane in the scene on which the extracted view will be focused is determined by the patch size: the larger the patch, the closer the focus plane. Advanced method allows smoothing the transitions between adjacent patches to reduce block artifacts. A gaussian weighted averaging (pixels that are further from the center have a smaller weight) is used to blend pixels outside the patches' borders. A disparity estimation method (based on block matching) proposed in [12] provides one estimated disparity value per MI depending on the depth of the objects inside each MI. This value (in pixels) corresponds to the adequate patch size to be used. This disparity-assisted patch blending extraction method (DAPBe [11]) provides full-focused extracted views, as each patch size is adapted to the depth of the content in the MIs.

\subsection{State-of-the-art integral image coding methods}

Integral images have a MIs based structure that involves a large number of grid-like edges (see Fig. 1). Additionally a large resolution is necessary to provide a large number of viewpoint images with a sufficient resolution. Therefore this kind of images are challenging to encode. A natural approach is to apply the Discrete Cosine Transform (DCT) to the MIs, followed by quantization and lossless coding [13]. Using the 3D-DCT on MIs stacked in 3D structures [14] can also exploit inter-MIs correlation. In [15], a Discrete Wavelet Transform (DWT) is applied to the MIs and a DCT is applied to the resulting blocks of coefficients (hybrid 4-dimensions

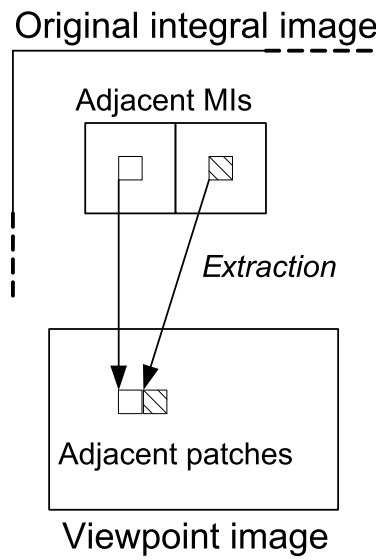

Figure 2. Patch based view extraction process 
transform). Although they fit the MIs based structure, transform-based approaches provide limited compression performances compared to current standard encoders (H.264/AVC [16] and HEVC [17]). In [18] and [19], several views are extracted and then encoded using MVC encoder [20]. This approach performs efficiently on computer generated images (i.e. that have MIs perfectly aligned on integer pixel values) but is limited for natural integral images. Non-local spatial correlation between MIs can be exploited by self-similarity (SS) modes [21] that are based on the principle of Intra Block Copy [22]. A block-matching algorithm is used similarly to the inter prediction modes of H.264/AVC and HEVC but within the current frame. Large compression gains are reported for still integral images but the performance is limited for sequences when temporal prediction is enabled.

A scalable coding scheme is proposed in [23] and described as follows: layer 0 corresponds to the central view, layer 1 corresponds to a set of additional views and layer 2 is the integral image. This layered scheme offers a display scalable feature (i.e. a stream that is adapted to 2D, to multi-view, and to light-field display systems). This scalability feature is however costly, as additional views are encoded. An inter-layer prediction method is therefore proposed where an integral image is sparsely reconstructed from the views (layer 1) and added to the reference frame list in order to reduce the bitrate of layer 2 .

We proposed an original coding scheme to encode integral images in [7], as described in Sec 3. Although it performs view extraction, and allows some kind of display scalability, it differs from existing methods: its main goal is compression efficiency. It takes advantages of the extraction process to reconstruct a reliable predictor and to create a residual integral image which is encoded.

\section{DESCRIPTION OF THE SCHEME AND PROPOSED IMPROVEMENTS}

\subsection{Compression scheme based on view extraction}

In [7] we proposed a compression scheme (Fig. 3 and Fig. 4) for integral images based on view extraction, described as follows. In this scheme, $\mathrm{II}_{\mathrm{R}}$ is the difference between the original image II and a reconstructed image II*. II* is reconstructed from viewpoint images extracted from the original integral image II. The residual integral image $\mathrm{II}_{\mathrm{R}}$ is encoded with HEVC (residual stream). Extracted views are encoded with 3D-HEVC (views stream). The number of views is not limited. Due to their small resolution, views represent a small number of bits to encode compared to II. Moreover, they have a natural image aspect that is less costly to encode than the MIs based structure of II. To obtain views with such a smooth aspect, advanced extraction methods are used, which use blending and varying patche sizes (see Sec. 2.1), both however preventing from perfect reconstruction with the exact original pixel values. The corresponding missing information, the difference between II and II*, is recovered in $\mathrm{II}_{\mathrm{R}}$. By definition, for a reconstructed image $\mathrm{II}^{*}$ close to the original II, the subtraction is expected to provide absolute values close to zero. Therefore $\mathrm{II}_{\mathrm{R}}$ has a flat aspect with low variations, which is easier to encode with HEVC than II.

During the reconstruction performed in this scheme, the patches in the viewpoint images are copied to their original position within the MIs in the reconstructed image $\mathrm{II}^{*}$, as illustrated in Fig. 5. Surrounding pixels contained in a zone of the same size as the MI (illustrated by the dotted squares) are also copied to fill the MIs in II*. These two steps are similar to the sparse reconstruction step and the micro-image refilling step described in [23]. Therefore, when reconstructing $\mathrm{II}^{*}$ from the extracted views, some missing pixels, coming from different

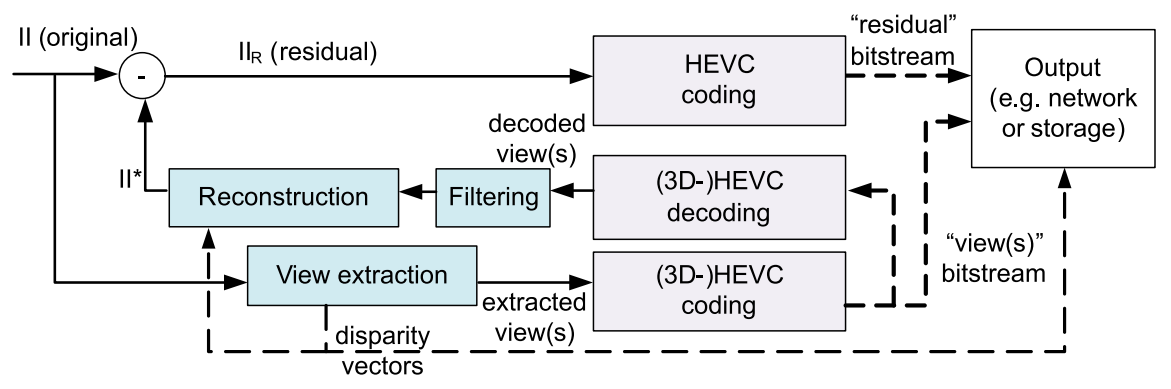

Figure 3. Proposed scheme - encoder side. 


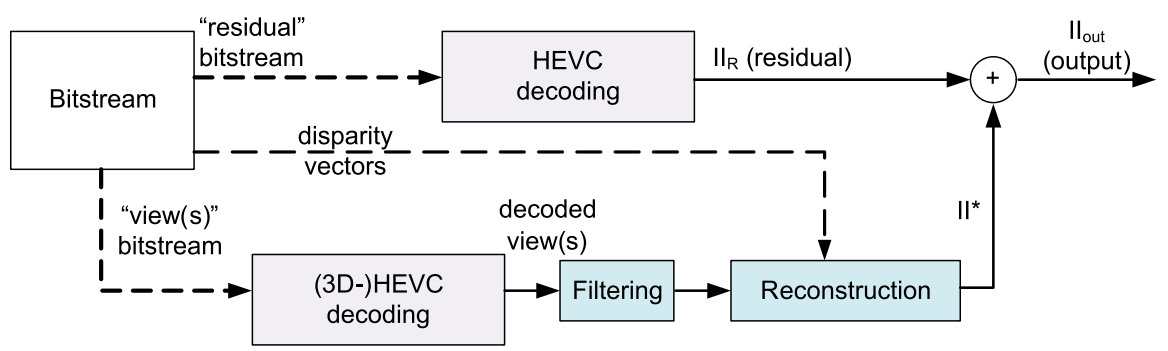

Figure 4. Proposed scheme - decoder side.

angles of view, are replaced by adjacent pixels from the same view (as illustrated in Fig. 5 with one view). However, the transformation of an object when changing the angle of view is not limited to a simple translation (disparity) but also involves angular differences. Hence errors are introduced. A low-pass filtering (e.g. average filter) is applied on the decoded views before the reconstruction to help smoothing these errors. High frequencies in the views are filtered while preserving the shape of the objects.

Disparity values computed at the extraction step are necessary for the reconstruction, and therefore are transmitted to the decoder, with the view(s) and the residual image $\mathrm{II}_{\mathrm{R}}$. At the decoder side (Fig. 4), the views are decoded and used to reconstruct $\mathrm{II}^{*}$, and $\mathrm{II}_{\mathrm{R}}$ is decoded and added to $\mathrm{II}^{*}$ to obtain the output image.

There is a tradeoff between rate and quality of the views and rate of $\mathrm{II}_{\mathrm{R}}$. $\mathrm{II}^{*}$ must be as close as possible to $\mathrm{II}$ in order to minimize the cost of $\mathrm{II}_{\mathrm{R}}$, without increasing too much the cost of the views. Several combinations are possible for the following parameters: the Quantization Parameter $(\mathrm{QP})$ used to encode the views $\left(\mathrm{QP} \mathrm{P}_{\mathrm{V}}\right)$, the $\mathrm{QP}$ used to encode the residual image $\left(\mathrm{QP} \mathrm{R}_{\mathrm{R}}\right)$, and the size (in pixels) of the average filter applied to the decoded view $(B)$. As in practice most of the bitrate is dedicated to $\mathrm{II}_{\mathrm{R}}$, the value of the parameter $\mathrm{QP}_{\mathrm{R}}$ is set according to the target bitrate (or quality), and $\mathrm{QP}_{\mathrm{V}}$ and $B$ are considered as parameters to optimize for a given $\mathrm{QP}_{\mathrm{R}}$. The number of extracted views and their positions (i.e. angle of view) also have an impact on the performance.

\subsection{Improvement with multi-view extraction}

In [7], only a single view is extracted and used to reconstruct II*. In this paper, we study the impact of different view extraction configurations on the compression performance. As described in Sec. 3.1, the efficiency of our scheme depends on a tradeoff between the bitrate of the views and the bitrate of the residual image. The goal here is to increase the number of extracted views in order to improve the reconstruction of $\mathrm{II}^{*}$, and therefore reduce the bitrate required to encode the residual image, without increasing too much the bitrate required for the views. Fig. 6 shows the relative positions of the patches within the MI for the tested configurations.

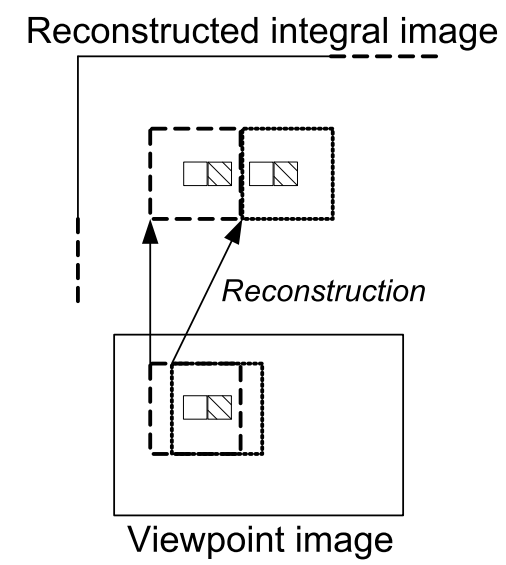

Figure 5. Reconstruction process from one view. 


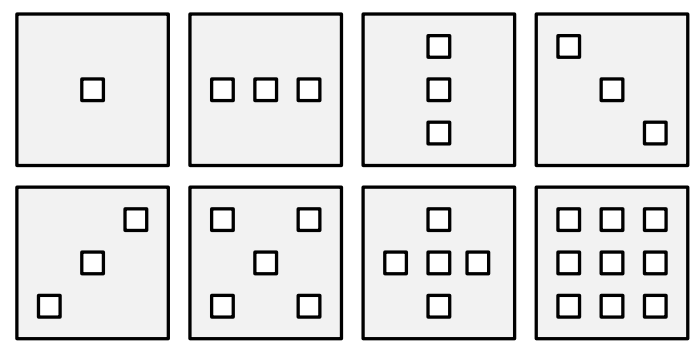

Figure 6. Examples of multi-view extraction configurations tested. Left-to-right, top-to-bottom: 1 view, 3 views horizontal, vertical, and diagonal, 5 views diagonal and straight, 9 views.

For the view extraction step in our experiments, we have implemented the disparity-assisted patch blending method (described in Sec. 2.1) according to [11]. However, in this work, the author only focuses and performs experiments on the most central point of view. The method has to be adapted to the extraction of side views. First, for the disparity estimation step, the adaptation basically consists in just shifting the position of the block used for the block matching algorithm, according to the target point of view. For the patch extraction step, pixels surrounding the borders of the patches are kept and blended with a weighted averaging, in order to avoid blocky artifacts in the view (see Sec. 2.1). As the patches have varying sizes (depending on the estimated disparity values), they are resized (with surrounding pixels in the MI) in order to match the maximum patch size. The distance from the patch to the center of the MI must be resized accordingly, so that the pixels used for the disparity estimation correspond to the pixels extracted in the patch, and to avoid a mismatch of angle of views between patches extracted from MIs with different disparity values.

Although blending the pixels surrounding the border of the patches reduces blocky artifacts, it can introduce another kind of artifact. For the side views that are far for the center, pixels close to the border of the MIs are included in the blending, introducing grid artifacts in the extracted views. Therefore in the following, we perform the experiments with views that are extracted with a smaller blending zone, presenting less artifacts as illustrated in Fig. 7.

\section{EXPERIMENTAL CONDITIONS}

Experimental conditions are as described in [7]. Experiments are performed on seven still integral images [8]. Incomplete MIs at the border and grid pixels corresponding to the boundaries of the micro-lenses [24] are removed.

Views are extracted using the disparity-assisted patch blending method [11] (see Sec. 2.1). Several configurations with 3, 5 and 9 views, as described in Sec. 3, are tested in order to be compared with the single view case. At the reconstruction of $\mathrm{II}^{*}$, for each MI, the pixels taken from all the views (within the patch and its surroundings) are all blended with an equal weight. In preliminary experiments, the reconstruction was performed by applying a gaussian weighted averaging centered on the patches, however these experiments provided a largely

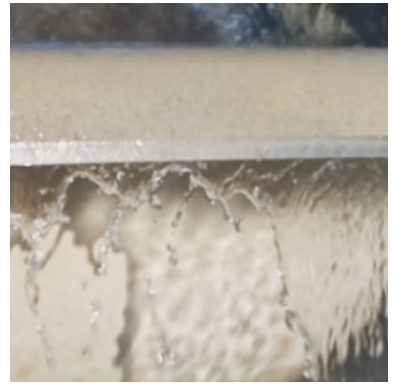

(a) central view, normal blending

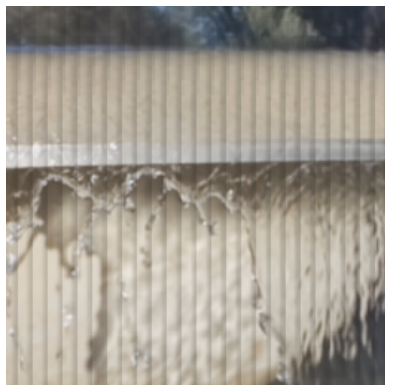

(b) side view, normal blending

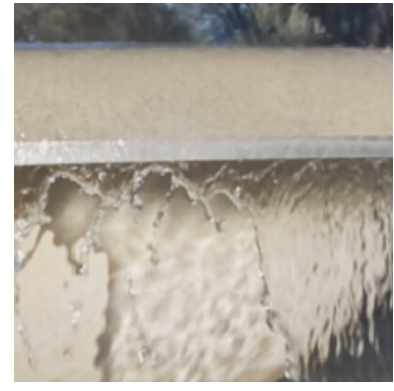

(c) side view, reduced blending

Figure 7. Views extracted at different positions with different blending zones (Fountain [8]) 
degraded compression performance. One possible reason for this unexpected result is that blending all the views smoothes the reconstruction unaccuracies and errors in the same way the low-pass filter does [7]. Preliminary experiments were also performed with 3 vertical views, in order to compare distances between the views in the range $\{5,10,15,20\}$ (in pixels). Although the results are image dependent, the distance 15 provided the better results in average. Therefore, a distance of 15 pixels between the views is used for the following experiments.

Views are encoded with 3D-HEVC reference software (HTM13.0). IPP inter-view prediction structure with the central view as base view is used in order to have the smallest distance possible between the reference and coding views as suggested in [25]. As the residual image can take values in the range [-255;+255], it is encoded with a bit depth of 10 bits. This encoding is performed under HEVC reference software (HM14.0) using the Intra main configuration [26]. Disparity values are coded with 4 bits per MI (1 value per MI in the range $\{1, \ldots, 15\})$. We already showed in [7] that a single value of $\mathrm{QP}_{\mathrm{V}}$ can be empirically associated to a value of $\mathrm{QP}_{\mathrm{R}}$ providing consistent gains (with a leave-one-out cross-validation method to select the experimental values). Therefore in this experiment, each tested $\mathrm{QP}_{\mathrm{R}}$ value in the range $\{10,15,20,25\}$ is associated to a $\mathrm{QP}_{\mathrm{V}}$ value in the range $\{20,22,25,31\}$. $B$ values providing the best results with one view are kept for each tested image. Compression results are provided using the Bjøntegaard Delta (BD) rate metric [27]. The anchor is II encoded with HEVC on the QP range $\{25,30,35,40\}$, and negative values represent improvement over the anchor.

\section{EXPERIMENTAL RESULTS}

Table 1. BD-rate results comparison between tested multi-view configurations.

\begin{tabular}{|l|c|c|c|c|c|c|c|c|}
\hline \multirow{2}{*}{ Image } & \multirow{2}{*}{1 view } & \multicolumn{4}{|c|}{3 views } & \multicolumn{2}{c|}{5 views } & \multirow{2}{*}{9 views } \\
\cline { 3 - 9 } & & ver. & hor. & \multicolumn{2}{|c|}{ diag. } & diag. & straight & \\
\hline Fountain & -17.0 & $-10,4$ & $-16,4$ & $-9,2$ & $-8,8$ & $-10,5$ & $-12,9$ & $-8,5$ \\
Fredo & -31.2 & $-29,1$ & $-28,9$ & $-27,4$ & $-26,0$ & $-22,6$ & $-27,9$ & $-22,0$ \\
Jeff & -5.4 & $-25,9$ & $-25,8$ & $-10,8$ & $-23,3$ & $-20,2$ & $-25,0$ & $-18,2$ \\
Laura & -11.1 & $-14,0$ & $-13,9$ & $-13,4$ & $-13,2$ & $-8,4$ & $-12,9$ & $-10,5$ \\
Seagull & -13.7 & $-13,4$ & $-13,7$ & $-13,3$ & $-13,3$ & $-8,0$ & $-11,1$ & $-10,9$ \\
Sergio & -23.5 & $-31,1$ & $-31,1$ & $-30,7$ & $-29,4$ & $-24,2$ & $-28,5$ & $-27,2$ \\
Zenhgyun1 & -7.1 & $-26,3$ & $-25,9$ & $-18,0$ & $-25,0$ & $-20,8$ & $-24,4$ & $-21,1$ \\
\hline Average & -15.6 & $-21,5$ & $-22,2$ & $-17,6$ & $-19,9$ & $-16,4$ & $-20,4$ & $-16,9$ \\
\hline
\end{tabular}

Tab. 1 shows the BD-rate results provided for each of the tested multi-view configurations. The configuration with 3 horizontal views provides the best results with an average BD-rate gain of $22.2 \%$ over the HEVC anchor. All the multi-view configurations overcome the performance of the single view configuration when considering the average BD-rate. However, some of them provide inconsistent results overall with significant losses for some images. For example the configuration with 3 vertical views is the second best in average but the BD-rate for Fountain drops from a $17 \%$ gain to a $10.4 \%$ gain only. Similarly, one of the 3 diagonal views configuration has a large average BD-rate gain of $19.9 \%$ but the gain for Fredo drops from $31.2 \%$ (which is our higher gain so far) to $26.0 \%$.

In our best configuration with 3 horizontal views, the improvement is quite consistent over the test set, with only slight losses reported for Fountain and Fredo (from $17.0 \%$ to $16.4 \%$ and from $31.2 \%$ to $28.9 \%$ respectively), similar results for Seagull and Laura (slight gain), and with large gains for the two images that provided the less impressive results with the single view configuration (from $5.4 \%$ to $25.8 \%$ for Jeff, and from $7.1 \%$ to $25.9 \%$ for Zenhgyun). Gains for Sergio are also significant (from $23.5 \%$ to $31.1 \%$ ). For images with large improvements, using one view is not sufficient to obtain an accurate reconstructed image, therefore significant angular information is contained in the residual image. Results show that this information is less costly when contained in two additional extracted views, providing a smoother reconstructed image with less errors. These results show that the increase of bitrate to encode several views can be compensated by the improvements of quality for II* and therefore the decrease of bitrate for $\mathrm{II}_{\mathrm{R}}$. 
Table 2. Fountain - Runtime variation against anchor, and percentage of the total time for each task including: extraction, reconstruction, view and residual encoding/decoding, and blur, subtraction and sum as others.

\begin{tabular}{|c|c|c|c|c|c|c|c|}
\hline & \multirow{2}{*}{ Runtime (\%) } & \multirow{2}{*}{$\begin{array}{l}\text { against } \\
\text { anchor }\end{array}$} & \multirow{2}{*}{ Extr. } & \multirow{2}{*}{ Rec. } & \multicolumn{2}{|c|}{ HEVC } & \multirow{2}{*}{ Others } \\
\hline & & & & & View & $\mathrm{II}_{\mathrm{R}}$ & \\
\hline \multirow{2}{*}{ Single view } & Encoding & 130 & 7 & 8 & 2 & 79 & 4 \\
\hline & Decoding & 240 & / & 31 & 1 & 46 & 22 \\
\hline \multirow{2}{*}{3 hor. views } & Encoding & 180 & 15 & 18 & 6 & 58 & 3 \\
\hline & Decoding & 390 & / & 57 & 1 & 28 & 14 \\
\hline
\end{tabular}

In some cases, even though the reconstruction of $\mathrm{II}^{*}$ is improved, the impact on the encoding of $\mathrm{II}_{\mathrm{R}}$ is not sufficient to compensate the additional cost of the views. For the image Seagull, using 3 vertical views improves the PSNR of II* against II (from $23.9 \mathrm{~dB}$ to $24.3 \mathrm{~dB}$ approximately) and provides a gain of $1.5 \%$ over the single view case when only the bitrate of the residual image $\mathrm{II}_{\mathrm{R}}$ is taken into account, but the gain drops to 0 when the bitrates of the views are included. In some other cases, like Fountain in the same 3 vertical views case, the improvement of the PSNR of II* against II (from $19.3 \mathrm{~dB}$ to $19.8 \mathrm{~dB}$ approximately) does not provide BD-rate gain, even without counting the views ( $5 \%$ loss approximately). This result shows that in these test conditions, the PSNR of II* against II is not as relevant as in the single view case [7] to predict the compression performance.

Table 2 provides the encoding and decoding runtime variations against the anchor (for Fountain), and the percentage of the runtime dedicated to each task. Encoding and decoding runtimes are respectively 1.3 and 2.4 times the anchor runtimes when using one extracted view [7]. In the multi-view case, only the runtime for the steps related to the views is impacted. Extraction and filtering consist basically of the same operations repeated for each views, therefore the runtime is multiplied by the number of views (i.e. 3 in our best configuration). Reconstruction runtime is also multiplied, although by slightly less than the number of views as some operations are common for all the views (e.g. normalization). Encoding and decoding times for the first coded view are the same as for one extracted view (as it is also an I frame). Additional runtime is required to encode side views (i.e. two $\mathrm{P}$ frames here). In our experimental conditions, encoding time for $\mathrm{P}$ frames is approximately 4 times larger than for I frames, while decoding time is similar. Total encoding and decoding time for the scheme using 3 horizontal views are respectively 1.8 and 3.9 times the anchor runtime.

\section{CONCLUSION}

In this paper we propose improvements for an integral image compression scheme. The original scheme proposed in [7] is based on the encoding of a residual integral image and an extracted view. The residual image results from the difference between the original integral image and an image reconstructed from the extracted view. Average BD-rate gains of $15.7 \%$ (up to $31.3 \%$ ) over the HEVC anchor have been previously reported when using one single extracted view, resulting in a realistic coding performance vs runtime codec. The efficiency of our scheme depends on a tradeoff between the bitrate of the views and the bitrate of the residual image. Hence we propose here to increase the number of extracted views in order to improve the quality of the reconstructed integral image, and therefore reduce the bitrate required to encode the residual image. Compression efficiency is increased with an average BD-rate gain of $22.2 \%$ (up to $31.1 \%$ ) over the HEVC anchor, at the cost of a realistic increase in runtime. In future work, the coding scheme will also be further evaluated with additional content and different methods (e.g. based on the quality of rendered views), and will be tested with different filtering for the view(s), with more advanced extraction methods using dense disparity maps, and with improved coding tools dedicated to the encoding of the residual image.

\section{ACKNOWLEDGMENTS}

Integral images included in our experiments are provided by Todor Georgiev [8]. 


\section{REFERENCES}

[1] Dufaux, F., Pesquet-Popescu, B., and Cagnazzo, M., [Emerging technologies for 3D video: content creation, coding, transmission and rendering], Wiley Eds (2013).

[2] Lippmann, G., "Epreuves reversibles donnant la sensation du relief," J. Phys. Theor. Appl. 7(1) (1908).

[3] Adelson, E. H. and Bergen, J. R., "The plenoptic function and the elements of early vision," Computational models of visual processing $\mathbf{1}(2)$ (1991).

[4] Lumsdaine, A. and Georgiev, T., "Full resolution lightfield rendering," Indiana University and Adobe Systems, Tech. Rep (2008).

[5] Tehrani, M. P., Senoh, T., Okui, M., Yamamoto, K., Inoue, N., and Fujii, T., "[m31095][FTV AHG] Use cases and application scenarios for Super Multiview Video and Free-Navigation," in [International Organisation For Standardisation], ISO/IEC JTC1/SC29/WG11 (2013).

[6] Tehrani, M. P., Senoh, T., Okui, M., Yamamoto, K., Inoue, N., and Fujii, T., "[m31103][FTV AHG] Introduction of Super Multiview Video systems for requirement discussion," in [International Organisation For Standardisation], ISO/IEC JTC1/SC29/WG11 (2013).

[7] Dricot, A., Jung, J., Cagnazzo, M., Pesquet, B., and Dufaux, F., "Integral images compression scheme based on view extraction," in [EUSIPCO], (2015).

[8] http://www.tgeorgiev.net/. Accessed: 2010-07-29.

[9] http://www.lytro.com/. Accessed: 2010-07-29.

[10] https://www.raytrix.de/. Accessed: 2010-07-29.

[11] Lino, J. F. O., "2D image rendering for 3D holoscopic content using disparity-assisted patch blending," Thesis to obtain the Master of Science Degree (2013).

[12] Georgiev, T. and Lumsdaine, A., "Focused plenoptic camera and rendering," Journal of Electronic Imaging 19(2) (2010).

[13] Forman, M., Aggoun, A., and McCormick, M., "Compression of integral 3D TV pictures," in [Fifth International Conference on Image Processing and its Applications], IET (1995).

[14] Forman, M. C., Aggoun, A., and McCormick, M., "A novel coding scheme for full parallax 3D-TV pictures," in $[I C A S S P], 4$, IEEE (1997).

[15] Elharar, E., Stern, A., Hadar, O., and Javidi, B., "A hybrid compression method for integral images using discrete wavelet transform and discrete cosine transform," Journal of display technology 3(3) (2007).

[16] Marpe, D., Wiegand, T., and Sullivan, G. J., "The H. 264/MPEG4 advanced video coding standard and its applications," Communications Magazine, IEEE 44(8) (2006).

[17] Sullivan, G. J., Ohm, J., Han, W.-J., and Wiegand, T., "Overview of the high efficiency video coding (HEVC) standard," Circuits and Systems for Video Technology, IEEE Transactions on 22(12) (2012).

[18] Dick, J., Almeida, H., Soares, L. D., and Nunes, P., "3D holoscopic video coding using MVC," in [EUROCON-International Conference on Computer as a Tool], IEEE (2011).

[19] Shi, S., Gioia, P., and Madec, G., "Efficient compression method for integral images using multi-view video coding," in $[I C I P]$, IEEE (2011).

[20] Ohm, J.-R., "Overview of 3D video coding standardization," in [International Conference on 3D Systems and Applications], (June 2013).

[21] Conti, C., Nunes, P., and Soares, L. D., "New HEVC prediction modes for 3D holoscopic video coding," in $[I C I P]$, IEEE (2012).

[22] Kwon, D.-K. and Budagavi, M., "Fast intra block copy (intraBC) search for HEVC screen content coding," in $[I S C A S]$, IEEE (2014).

[23] Conti, C., Nunes, P., and Soares, L. D., "Inter-layer prediction scheme for scalable 3-D holoscopic video coding," Signal Processing Letters 20(8) (2013).

[24] Georgiev, T., Zheng, K. C., Curless, B., Salesin, D., Nayar, S., and Intwala, C., "Spatio-angular resolution tradeoffs in integral photography.," Rendering Techniques (2006).

[25] Dricot, A., Jung, J., Cagnazzo, M., Pesquet, B., and Dufaux, F., "Full parallax super multi-view video coding," in $[I C I P]$, IEEE (2014).

[26] Bossen, F., "Test conditions and software reference configurations," JCT-VC L1100 (2013).

[27] Bjøntegaard, G., "Calculation of average PSNR differences between RD-curves," in [VCEG Meeting], (2001). 